Ophthalmologe 2022 $\cdot 119: 1149-1151$ https://doi.org/10.1007/s00347-022-01578-7 Eingegangen: 24. Dezember 2021 Überarbeitet: 1. Januar 2022 Angenommen: 9. Januar 2022 Online publiziert: 28. Januar 2022 (c) Der/die Autor(en) 2022

\section{Overlap-Syndrom aus Riesenzellarteriitis und Pachymeningitis}

\author{
Carolin Kolb (DiD - Michael Müller - Thomas Kohnen \\ Klinik für Augenheilkunde, Universitätsklinikum Frankfurt, Goethe-Universität Frankfurt, Frankfurt am \\ Main, Deutschland
}

Erstautorin:Dr.med.Carolin Kolb

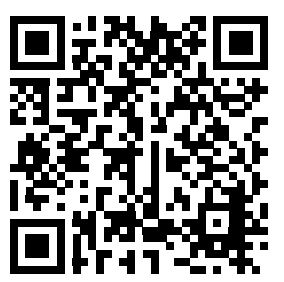

QR-Code scannen \& Beitrag online lesen

\section{Anamnese}

Ein 81-jähriger Patient berichtete, dass er seit etwa 4 Wochen einen leichten Schleier vor dem rechten Auge sehe, welcher über Nacht aufgetreten sei. $\mathrm{Er}$ habe zudem neu aufgetretene frontale Kopfschmerzen insbesondere im Liegen. Diese hätten einen drückenden Charakter und seien während des Tages geringer ausgeprägt als in der Nacht. Zur Abklärung sei bereits 3 Wochen zuvor eine kraniale Computertomographie erfolgt, die keinen pathologischen Befund ergeben habe. Seit 1 Woche habe der Patient eine massive Sehverschlechterung auch am linken Auge bemerkt. Der Patient verneinte Schmerzen am Auge sowie Kau-, Kämm-, Schläfen- oder Muskelschmerzen. Internistische Vorerkrankungen waren nicht bekannt.

\section{Befund}

Bei Erstvorstellung betrug der Visus Handbewegungen beidseits. Die Bulbusmotilität war frei und ohne Bulbusbewegungsschmerz. Ein relativer afferenter Pupillendefekt bestand nicht. Die Aa. temporales superficiales waren seitengleich gut tastbar, ohne Verdickung oder Druckschmerzhaftigkeit. Die Tensiowerte waren mit 14 und $11 \mathrm{mmHg}$ normwertig. In der klinischen Untersuchung der Vorderabschnitte zeigte sich beidseits eine reizfreie Pseudophakie. Ophthalmoskopisch war die Papille beidseits randscharf, vital und physiologisch exkaviert. Makula, Gefäße sowie zentrale und periphere Netzhaut waren unauffällig. Die optische Kohärenztomographie von Papille und Makula waren regelrecht. In der Perimetrie ergab sich rechts eine konzentrische Gesichtsfeldeinengung. Links waren die Außengrenzen nasal betont reduziert (• Abb. 1). Die neurologische Untersuchung war mit Ausnahme der Visusminderung unauffällig. In der kranialen Magnetresonanztomographie (cMRT) fielen nebenbefundlich schmale chronische Subduralhämatome auf ohne raumfordernden Effekt und Interventionsbedarf. Hinweise auf einen akuten oder abgelaufenen Infarkt ergaben sich nicht, ebenso keine Stenosen der Hirnbasisarterien.

Mit der Arbeitsdiagnose einer Durchblutungsstörung unklarer Genese wurde der Patient zur weiteren Diagnostik und Therapie stationär aufgenommen. Im Aufnahmelabor zeigten sich eine erhöhte Erythrozytensedimentationsrate (ESR) von $96 \mathrm{~mm} / \mathrm{h}$ sowie ein erhöhtes C-reaktives Protein (CRP) von 17,3 mg/l, sodass aufgrund des Verdachtes auf eine arteriitische Komponente eine intravenöse Therapie mit $500 \mathrm{mg}$ Prednisolon täglich gestartet wurde.

Eine erneute cMRT mit Angiographie ergab keinen Hinweis auf eine Vaskulitis der extra- oder intrakraniellen Gefäße. Es zeigte sich jedoch eine rechtsbetonte Duraverdickung mit Kontrastmittelanreicherung (- Abb. 2), die radiologisch am ehesten als immunvermittelt angesehen wurde. Aufgrund der Differenzialdiagnose einer Pachymeningitis wurde eine Liquorpunktion vorgenommen, in der sich ein entzündlicher Liquor (Leukozyten 19/ $\mu$, Gesamtprotein $597 \mathrm{mg} / \mathrm{l}$ ) mit mononukleärem Zellbild fand. Die Multiplex-Polymerasekettenreaktion (PCR) auf Bakterien und Viren war negativ, ebenso die Liquorkulturen. Die farbcodierte Dopplersonographie der extrakraniellen Gefäße zeigte rechts 


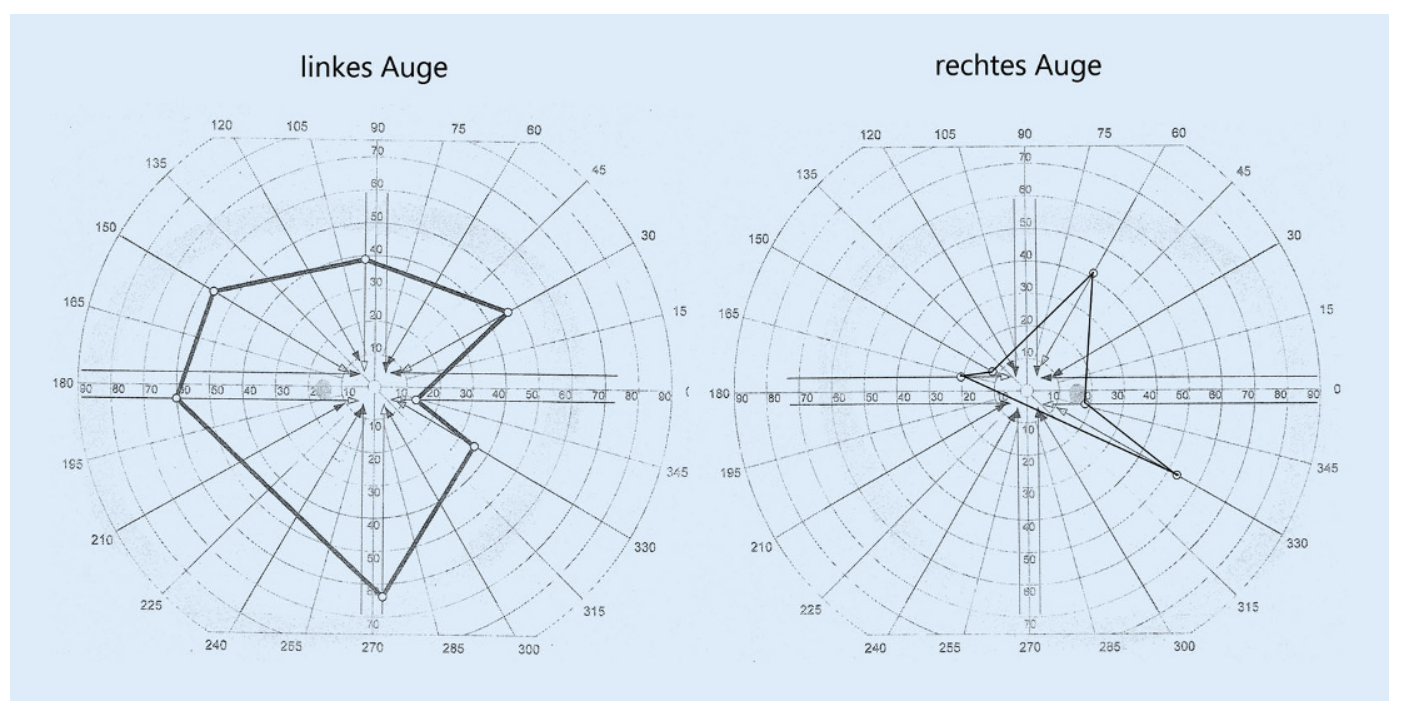

Abb. $1 \varangle$ Perimetrie mit konzentrischer Gesichtsfeldeinengung rechts und nasal betonter Reduktion der Außengrenzen links

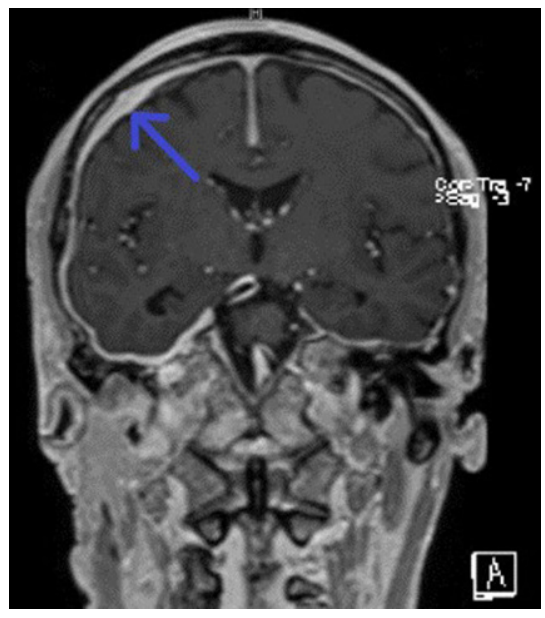

Abb. $2 \Delta$ cMRT mit rechtsbetonter Duraverdickung (siehe Pfeil) und Kontrastmittelanreicherung im Rahmen einer hypertrophen Pachymeningitis

ein Halo-Zeichen als Hinweis auf eine Vaskulitis der A. temporalis ( $\mathbf{A b b}$. 3).

Die kardiologische Abklärung ergab unauffällige Befunde in der 24-h-Blutdruckmessung, dem Elektrokardiogramm und der transthorakalen Echokardiographie.

\section{Diagnose}

Overlap-Syndrom aus Riesenzellarteriitis (RZA) mit Arteriitis temporalis rechts und immunvermittelter hypertropher Pachymeningitis (HP).

\section{Therapie und Verlauf}

Bereits am ersten Tag nach Therapiebeginn betrug der Visus mit eigener Korrektur (ccs)
0,5 beidseits. Die ESR sank auf $46 \mathrm{~mm} / \mathrm{h}$ am zweiten und $35 \mathrm{~mm} / \mathrm{h}$ am fünften Tag des stationären Aufenthaltes. Nach 5 Tagen intravenöser Therapie mit Prednisolon $500 \mathrm{mg}$ wurde die Dosis auf $100 \mathrm{mg}$ täglich reduziert. Aufgrund einer steroidinduzierten arteriellen Hypertonie wurde der Patient zur Einleitung einer steroidsparenden Therapie auf die rheumatologische Station verlegt. Bei Verlegung betrug der Visus ccs 0,9 beidseits sowie ccs 1,0 binokular. Die Kopfschmerzen hatten sich ebenfalls deutlich gebessert. Bei der augenärztlichen Kontrolle 1 Monat nach Erstvorstellung war das Sehvermögen subjektiv stabil. Kopfschmerzen wurden keine mehr angegeben. Der Patient beklagte jedoch weiterhin eine erhöhte Blendempfindlichkeit, welche er als mäßig störend im Alltag empfand. Die Prednisolon-Dosis wurdealle 2 Wochen um $10 \mathrm{mg}$ reduziert. Gleichzeitig wurde eine Therapie mit Tocilizumab subkutan 1-mal pro Woche eingeleitet. In der Kontrolle nach 3 Monaten ergab sich kein Hinweis auf ein Rezidiv bei weiterhin bestehender Blendempfindlichkeit.

\section{Diskussion}

Laut aktueller Leitlinie zum Management der Großgefäßvaskulitiden muss die Diagnose einer RZA durch die Zusammenschau aller Befunde gestellt werden, da kein "trennscharfer diagnostischer Biomarker" zur Verfügung steht [8]. Eine Biopsie der A. temporalis wurde lange als diagnostischer Goldstandard angesehen. Sie ist jedoch der Sonographie bezüglich der Sen- sitivität unterlegen und daher nicht mehr primär empfohlen [1]. Da bei dem geschilderten Patienten klinische und laborchemische Hinweise auf eine RZA vorlagen, in der Dopplersonographie ein Halo-Zeichen als Hinweis auf eine Arteriitis temporalis sichtbar war und es unter der Therapie zu einer raschen Visusverbesserung kam, wurde auf die invasive Diagnostik verzichtet.

Bei Verdacht auf eine RZA ist eine sofortige Hochdosissteroidtherapie erforderlich. Die Empfehlungen hinsichtlich der Dosierung variieren je nach Fachgesellschaft und Land zwischen 250 und $1000 \mathrm{mg}$ [7]. Steroidsparende Therapien, beispielsweise mit dem Interleukin-6-Rezeptor-Antikörper Tocilizumab, sind meist im Verlauf erforderlich [9].

Die HP ist eine seltene Erkrankung, bei der es zu einer entzündlichen Verdickung der Dura mater kommt. Sie tritt in etwa der Hälfte der Fälle idiopathisch auf. Sekundäre Ursachen sind systemische inflammatorische Erkrankungen wie Sarkoidose, Granulomatose mit Polyangiitis, Sjögren-Syndrom, rheumatoide Arthritis oder IgG4-assoziierte Erkrankung. Maligne Prozesse oder Infektionen wie Tuberkulose, Lues oder Borreliose können ebenso ursächlich sein $[3,6]$. Klinisch sind die Patienten asymptomatisch oder fallen mit Kopfschmerzen, Visusverlust und Hirnnervenausfällen auf. Seltener treten Doppelbilder oder ein Papillenödem auf [6]. Die radiologischen Befunde sind meist unspezifisch und liefern nur geringe Hinweise auf die Genese der Erkrankung. So zeigt die 


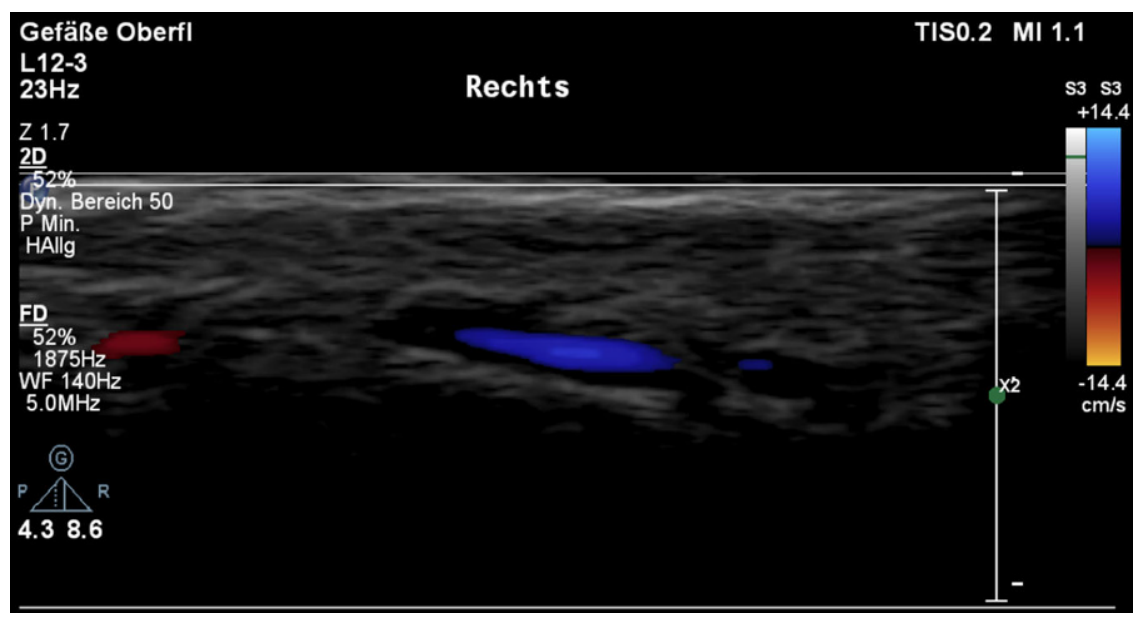

Abb. 3 ॥ Farbcodierte Dopplersonographie der rechten A. temporalis mit echoarmer Wandverdickung (Halo) als Hinweis auf eine Arteriitis temporalis

idiopathische HP eher eine diffuse Kontrastmittelanreicherung, wohingegen bei Sarkoidose eher eine noduläre Anreicherung zu erwarten ist [3]. Laborchemisch ist meist eine ESR-Erhöhung, seltener eine CRP-Erhöhung auffällig. In der Hälfte der Fälle findet man erhöhte Proteinkonzentrationen und eine Lymphozytose im Liquor, sodass an die Differenzialdiagnose einer aseptischen Meningitis gedacht werden muss [6]. Im Falle unseres Patienten erschien eine immunvermittelte Pachymeningitis aufgrund des raschen Ansprechens auf die Steroidtherapie jedoch deutlich wahrscheinlicher. Zudem waren die Ergebnisse der Virus-PCR im Verlauf negativ. Da die Befunde der nicht- oder minimal-invasiven Diagnostik in vielen Fällen einer HP unspezifisch sind, ist eine Biopsie der Dura mater zum Nachweis der Entzündungsreaktion und Ausschluss von Differenzialdiagnosen manchmal nicht zu umgehen [3]. Die Symptome einer hypertrophen Pachymeningitis sind unter Steroidtherapie zwar regredient, eine komplette Symptomfreiheit wird allerdings selten erreicht. Zudem kommt es bei Steroidreduktion häufig zu einem Rezidiv. Nach einer erneuten hoch dosierten Steroidgabe ist dann in diesen Fällen eine dauerhafte Immunsuppression erforderlich [6].

Es gibt wenige beschriebene Fälle in der Literatur, in denen ein Overlap-Syndrom aus RZA und HP aufgetreten ist [7]. Die HP kann dabei nicht nur simultan, sondern auch mehrere Jahre nach der Erstdiagnose einer RZA auftreten [2]. Ursächlich für die Verdickung und Kontrastmittelan- reicherung der Dura scheint eine Leckage der duraversorgenden $A$. meningea media im Rahmen der Vaskulitis zu sein [4]. Die Kopfschmerzen werden eher auf die Duraverdickung als auf die RZA zurückgeführt [5].

\section{Fazit für die Praxis}

- Insbesondere bei sonographisch unauffälligen Temporalarterien sollte bei Visusminderung, Kopfschmerzen und ESR-Erhöhung an eine HP gedacht werden und bei Verdacht ein cMRT erfolgen. Therapeutisch stehen Steroide im Vordergrund.

- Ebenso wie bei der RZA ist bei der HP eine enge Zusammenarbeit mit den neurologischen und rheumatologischen Fachabteilungen nötig, da eine dauerhafte Therapie erforderlich ist.

- RZA und HP können auch gemeinsam als Overlap-Syndrom auftreten.

\section{Korrespondenzadresse}

Univ.-Prof. Dr. med. Thomas Kohnen Klinik für Augenheilkunde, Universitätsklinikum Frankfurt, Goethe-Universität Frankfurt Theodor-Stern-Kai 7, 60590 Frankfurt am Main, Deutschland

kohnen@em.uni-frankfurt.de

Funding. Open Access funding enabled and organized by Projekt DEAL.

\section{Einhaltung ethischer Richtlinien}

Interessenkonflikt. M. Müller geht einer Vortragstätigkeit für Alcon/Novartis, Allergan, Thea nach. T. Kohnen gibt Forschungsförderung und Beratung für Alcon/Novartis, Avedro, J\&J, LensGen, Oculentis,
Oculus, Presbia, Schwind, Zeiss, Berater für Allergan, Bausch \& Lomb, Dompé, Geuder, med update, Nevarkar, Santen, Staar, Tear Lab, Thieme, Ziemer an. C. Kolb gibt an, dass kein Interessenkonflikt besteht.

Für diesen Beitrag wurden von den Autoren keine Studien an Menschen oder Tieren durchgeführt. Für die aufgeführten Studien gelten die jeweils dort angegebenen ethischen Richtlinien. Für Bildmaterial oder anderweitige Angaben innerhalb des Manuskripts, über die Patienten zu identifizieren sind, liegt von ihnen und/oder ihren gesetzlichen Vertretern eine schriftliche Einwilligung vor.

Open Access. Dieser Artikel wird unter der Creative Commons Namensnennung 4.0 International Lizenz veröffentlicht, welche die Nutzung, Vervielfältigung, Bearbeitung, Verbreitung und Wiedergabe in jeglichem Medium und Format erlaubt, sofern Sie den/die ursprünglichen Autor(en) und die Quelle ordnungsgemäß nennen, einen Link zur Creative Commons Lizenz beifügen und angeben, ob Änderungen vorgenommen wurden.

Die in diesem Artikel enthaltenen Bilder und sonstiges Drittmaterial unterliegen ebenfalls der genannten Creative Commons Lizenz, sofern sich aus der Abbildungslegende nichts anderes ergibt. Sofern das betreffende Material nicht unter der genannten Creative Commons Lizenz steht und die betreffende Handlung nicht nach gesetzlichen Vorschriften erlaubt ist, ist für die oben aufgeführten Weiterverwendungen des Materials die Einwilligung des jeweiligen Rechteinhabers einzuholen.

Weitere Details zur Lizenz entnehmen Sie bitte der Lizenzinformation auf http://creativecommons.org/ licenses/by/4.0/deed.de.

\section{Literatur}

1. Bardi M, Diamantopoulos AP (2019) EULAR recommendations for the use of imaging in large vessel vasculitis in clinical practice summary. Radiol Med 124:965-972

2. Boisch G, Duda S, Hartmann C, Weßling H (2018) Hypertrophic pachymeningoencephalitis associated with temporal giant cell arteritis. BMJ Case Rep 2018:bcr-2018-225304

3. Hahn LD, Fulbright R, Baehring JM (2016) Hypertrophic pachymeningitis. J Neurol Sci 367:278-283

4. Joelson E, Ruthrauff B, Ali F et al (2000) Multifocal dural enhancement associated with temporal arteritis. Arch Neurol 57:119-122

5. Kuhn J, Harzheim A, Brockmann Metal (2004) Focal hypertrophic pachymeningitis in association with temporal arteritis. Headache 44:1045-1048

6. Kupersmith MJ, Martin V, Heller G et al (2004) Idiopathic hypertrophic pachymeningitis. Neurology 62:686-694

7. Kutty RK, Maekawa M, Kawase T et al (2020) Temporal arteritis with focal pachymeningitis: a deceptive association. Nagoya J Med Sci 82:143-150

8. Schirmer JH, Aries PM, Balzer K et al (2020) S2kLeitlinie:Management derGroßgefäßvaskulitiden. ZRheumatol 79:67-95

9. Stone JH, Tuckwell K, Dimonaco S et al (2017) Trial of tocilizumab in giant-cell arteritis. N Engl J Med 377:317-328 